\title{
La experimentación científica en Educación Inicial
}

\author{
The scientific experimentation in Initial Education \\ Fraismar Castillo \\ fraismar84@yahoo.com \\ Código ORCID: 0000-0002-8350-0515 \\ Jardín de Infancia Rómulo Gallegos, Miranda, Venezuela
}

Recibido: Abril 2019 / Arbitrado: Mayo 2019 / Publicado: Julio 2019

RESUMEN

La presente investigación se planteó promover la experimentación científica en los niños de Educación Inicial, a partir del diseño de estrategias de enseñanza que fueran innovadoras. La metodología empleada fue bajo un enfoque cualitativo fundamentado en una investigación de campo de carácter descriptivo - interpretativo. Los sujetos fueron dos docentes y 31 niños del primer grupo del Preescolar Ambrosio Plaza, ubicado en Guarenas, estado Miranda, Venezuela. Los resultados evidenciaron que los docentes en esta institución imparten sus clases de manera tradicional, esto es por áreas de aprendizaje para que los niños ejecuten alguna actividad cotidiana. De allí que se diseñaran unas estrategias de enseñanza que mostraran una estructura metodológica de pedagogía activa para promover la exploración y experimentación del entorno natural de los niños del preescolar. Todo esto resultará altamente efectivo por cuanto se despertará el interés, la motivación y la curiosidad de los niños.

Palabras clave: Estrategias de enseñanza; promoción; experimentación científica; preescolar 


\section{INTRODUCCIÓN}

En esta época de grandes y acelerados cambios, se requiere de individuos bien formados con habilidades cognitivas desarrolladas que les permitan ser altamente competitivos, participativos, autónomos, responsables y creativos; pero que también tengan capacidades para resolver problemas y enfrentarse a experiencias significativas que enriquezcan su desarrollo.

Por ello, es necesaria una educación actualizada y preocupada por incorporar estrategias innovadoras que contribuyan con la formación del individuo que hoy día exige la sociedad. Desde los primeros grados de escolaridad, el niño debe estar inmerso en procesos de enseñanza y aprendizaje ajustados a experiencias reales y que despierten su curiosidad y su pensamiento reflexivo.

En el documento de Educación Inicial. Bases Curriculares (Ministerio de Educación y Deportes, 2005) se establece que "el paradigma educativo que se construye en el país tiene como centro al ser humano como ser social, capaz de responder y participar activamente en la transformación de la sociedad en la que vive y se desarrolla" (p. 5). Por ello, la Educación Inicial necesita docentes conocedores del sistema educativo, que planifiquen y ejecuten estrategias de enseñanza cónsonas con los aprendizajes necesarios para cada niño.

Rico (2000) concibe que: “... con frecuencia se exige al maestro, en la práctica escolar, trabajar para una enseñanza desarrolladora, esto es trabajar para el desarrollo de las potencialidades de sus alumnos" (p. 2). Por lo que las estrategias por parte del maestro tienen que estar bien sustentadas en un diagnóstico y en una evaluación de los aprendizajes de los niños, además de contextualizar que no todos tienen los mismos intereses ni las mismas necesidades y que cada uno va a su propio ritmo para aprender. De allí que el maestro debe buscar las estrategias de enseñanza acordes con las necesidades e intereses de los educandos para que de esta forma ocurra de manera significativa el aprendizaje.

Díaz Barriga y Hernández (2010) describen que: “... las estrategias de enseñanza son procedimientos que el agente de enseñanza utiliza en forma reflexiva y flexible para promover el logro de aprendizajes significativos en los alumnos" (p.118). Las estrategias de enseñanza, o como Alfonzo (2003) las denominan estrategias instruccionales, son pues las que utiliza el docente como medio principal para su planificación, con la única finalidad de que los niños puedan apropiarse del conocimiento y así ellos logren interesarse en descubrirlo.

Las afirmaciones anteriores dejan claro dos aspectos: el interés de utilizar las estrategias de enseñanza en el nivel de Educación Inicial y que los maestros deben conocer y manejar las estrategias para integrar los nuevos aprendizajes, tal es el caso de la ciencia en el preescolar.

Delors (1997) sostiene que es de gran importancia introducir la alfabetización científica desde el nivel de Educación Inicial, enumerando una variedad de razones tales como: a) que contribuye con la formación de problemas lógicos a través de la resolución de problemas concretos, b) que mejora la calidad de vida y c) que prepara al niño para la futura inserción en el mundo científico tecnológico.

Por su parte, Serrano (2008) sostiene que los niños pueden aprender ciencias en formas diversas y lo pueden hacer más fácilmente cuando el aprendizaje surge a partir de la satisfacción de sus propias necesidades e intereses. En consecuencia, la enseñanza de las ciencias es importante en tanto que lleve a los niños a reflexionar y les brinde la satisfacción que implique el descubrir mediante la experimentación (p. 131).

Algunos autores como Gallegos et al. (2008) afirman que son poco frecuentes las investigaciones sobre las ideas de los procesos y conceptos de ciencias que tienen los niños, cuyas edades oscilan entre los 3 y los 6 años de edad y, en este sentido, la mayoría de las veces los 
procesos de enseñanza propuestos en esta área escasamente toman en cuenta los intereses de los educandos.

En relación con los supuestos anteriores, se puede evidenciar que en Educación Inicial se debe fomentar la ciencia desde los primeros años en edad escolar. No obstante, en el caso del Preescolar Ambrosio Plaza, los niños no estaban logrando aprendizajes significativos de la ciencia, específicamente en el espacio de experimentar y descubrir, el cual permite satisfacer las necesidades que tienen los niños de sentir, oler, tocar, explorar, manipular, formular hipótesis, comprobar entre otras, tal como lo señala el documento Educación Inicial. Bases Curriculares (Ministerio de Educación, 2005). De allí que el presente estudio se planteó el siguiente objetivo: promover la experimentación científica en los niños del Preescolar Ambrosio Plaza, ubicado en Guarenas, estado Miranda- Venezuela, a partir del diseño de estrategias de enseñanza innovadoras.

\section{Estrategias de enseñanza}

La renovación educativa implica la definición de nuevas herramientas aplicables a los modelos actuales de la enseñanza y aprendizaje. En este sentido, cabe señalar que para encontrar un nuevo horizonte educativo en el cual los niños aprendan a aprender y sean los protagonistas del desarrollo de su propio conocimiento, se hace necesario que el docente de educación inicial asuma el uso de nuevas estrategias, para lograr tal cometido en la enseñanza de la ciencia.

Para Uría (2001) la estrategia hace referencia al proceso que envuelve los eventos pedagógicos; es decir, las técnicas, las actividades y los medios utilizados para la enseñanza y el aprendizaje. Beltrán (1996) afirma que: "las estrategias tienen un carácter intencional; implican, por tanto, un plan de acción, frente a la técnica, que es marcadamente mecánica y rutinaria" (p. 394).

Por su parte, Díaz y Hernández (2010) manifiestan que la enseñanza es en gran medida una autentica creación: “... una actividad que consiste en una constante lectura de la situación y que exige no ser abordada a través de una mirada estrictamente técnica, sino por lo contrario una actividad reflexiva y estratégica" (p. 117). Así, el desarrollo del proceso de enseñanza supone la lectura, el conocimiento y entrenamiento del docente, lo que permitirá abordar de una forma novedosa y distinta la actividad a realizar.

Las estrategias de enseñanza constituyen los medios que dispone el docente para lograr construir en los alumnos aprendizajes significativos en la enseñanza de la ciencia. Por tal razón, el maestro de educación inicial debe hacer un buen manejo de estas, saber qué función tiene, cuáles son las más adecuadas para enseñar cada situación específica y cómo pueden utilizarse o desarrollarse apropiadamente para lograr el objetivo.

El adecuado uso de las estrategias de enseñanza lleva a una instrucción estratégica interactiva y de alta calidad. Según Beltrán (1996) el instructor estratégico debe ser un mediador y un modelo para el estudiante. El docente debe dirigir su acción a influir en los procesos de aprendizaje. La clasificación de las estrategias de enseñanza dependerá según el momento de uso o de su presentación y pueden ser descritas de la siguiente manera:

Preinstruccionales: preparan al estudiante en relación a qué y cómo va a aprender, incidiendo en la activación o generación de conocimientos previos y ubicándolo en el contexto conceptual pertinente. Algunas de las estrategias más típicas son los objetivos y los organizadores previos.

Coinstruccionales: apoyan los contenidos durante el desarrollo del proceso de enseñanza y aprendizaje, para que el estudiante mejore la atención, codifique y conceptualice los contenidos. Aquí pueden incluirse estrategias como ilustraciones, preguntas intercaladas, señalizaciones, redes y mapas conceptuales, analogías y cuadros C$\mathrm{Q}-\mathrm{A}$, entre otras. 
Posinstruccionales: se muestra el término de la situación de enseñanza y permite formar una visión sintética, integradora del material, valorando todo lo aprendido durante el proceso. Algunas de las estrategias más reconocidas en este momento son resúmenes finales, organizadores gráficos (cuadros sinópticos simples y de doble columna), redes y mapas conceptuales.

En la práctica docente, los tres momentos descritos mantienen su orden, es decir siempre habrá un inicio, un desarrollo y un cierre de la sección de enseñanza y aprendizaje; igualmente, es necesario resaltar que cada momento instruccional se constituye por eventos instruccionales, los cuales son el epicentro que genera una comunicación didáctica desarrolladora entre el agente de enseñanza y el agente de aprendizaje (Feo, 2010).

Es preciso indicar que el docente de educación inicial deba adaptar las estrategias a las necesidades de los niños y a los objetivos que aspira concretar, siendo el caso particular de la experimentación científica. En suma el docente debe planear un método que se amolde a los educandos y así, disminuir las diversas dificultades en el aprendizaje que puedan suscitarse, transformándolas en productividad y asegurando éxito en cada acción pedagógica.

Estas se van a transformar siguiendo una planificación y actividades innovadoras y significativas, estas se pueden representar dentro y fuera del aula. Los espacios no deben limitarse para que así el niño logre entender que la ciencia esta en cualquier lugar de su entorno.

\section{El currículo de Educación Inicial}

La Educación Inicial es la primera etapa de la Educación Bolivariana y está dirigida a la población entre 0 y 6 años o hasta su ingreso al primer grado de Educación Básica, con el fin de garantizar una educación integral de calidad, permanente, en igualdad de condiciones y de oportunidades; a través de la atención convencional y no convencional, con la participación de la familia y la comunidad (Ministerio de Educación y
Deportes, 2007). Tiene como propósito la formación integral de los niños, en cuanto a hábitos, habilidades, destrezas, actitudes y valores basados en la identidad local, regional y nacional, mediante el desarrollo de sus potencialidades y el pleno ejercicio de sus derechos como persona en formación, atendiendo a la diversidad e interculturalidad.

Se administra en dos niveles: una maternal, para niños desde la gestación hasta cumplir los tres años y la fase preescolar, dirigida a la población de tres a seis años.

El nivel maternal se refiere a la educación integral de los niños en la cual la familia y especialmente la madre, cumplen un papel fundamental, considerando las características de desarrollo y las necesidades de este grupo etéreo, especialmente las de afecto y comunicación. Igualmente, un elemento importante en esta fase de vida es que los niños necesitan el contacto humano físico, la relación madre-hijo o hija, para establecer el vínculo que permitirá el desarrollo social y emocional. Ese vínculo o apego constituye el primer lazo social que se desarrolla entre madre e hijo, base de la socialización del ser humano (Ministerio del Poder Popular para la Educación Inicial, 2014).

El nivel preescolar se orienta a los niños desde los 3 años hasta cumplir los 6 años o hasta su ingreso a primer grado de Educación Básica, al igual que el nivel maternal ofrece atención en instituciones educativas, en la familia y en la comunidad. Se continúa con la atención integral de los educandos, fortaleciendo el Área Pedagógica ejecutada por distintos actores educativos 0 personas significativas, que promueven experiencias de aprendizaje que faciliten el desarrollo pleno de sus potencialidades, para que puedan encarar con éxito la escolarización de la Educación Básica (Ministerio del Poder Popular de Educación Inicial, 2014).

\section{La enseñanza de la ciencia en el preescolar}

Según Del Niño y Maldonado (2007), en el preescolar la enseñanza de la ciencia requiere 
conocer al niño y orientarlo en la búsqueda de respuestas de todo aquello que lo inquieta en su entorno. Es importante considerar que el niño toma conciencia del mundo físico y biológico que lo rodea, a partir de la observación y de la exploración del medio ambiente inmediato.

A los tres años, aparecen los porqué, al no perseguir la causa mecánica de los fenómenos, el cómo no le interesa y sus porqué son indiferenciados; estos no tienen como propósito descubrir la causa final de los hechos, porque al no comprender las causas físicas de los mismos, él se los explica mágicamente. Es propio del período preoperatorio el animismo, el cual resulta de una confusión o disociación entre su mundo interior o subjetivo y el universo físico. Al creer que los objetos de la naturaleza son seres animados, les atribuye vida, conciencia, voluntad e intencionalidad.

Aparece luego la tendencia a considerar los fenómenos físicos como producto de la creación humana; el niño cree que los objetos y fenómenos que lo rodean fueron hechos por los seres humanos con propósitos específicos; atraviesa por la etapa del artificialismo (el ser humano es capaz de hacer mover las nubes); confusión del mundo interior y exterior provocada por su egocentrismo. Más adelante la reducción gradual del egocentrismo lo lleva a la socialización progresiva del pensamiento. El niño descubre que los otros no piensan como él; se adapta a nuevas situaciones y reemplaza la lógica egocéntrica por la lógica verdadera obligada por su vida en sociedad.

El docente debe gradualmente poner al alcance de los niños materiales y elementos que le permitirán descubrir el porqué de los hechos y fenómenos de la naturaleza, dar oportunidad de observar, preguntar, explorar y experimentar, en razón directa con los intereses infantiles y de acuerdo con una planificación bien meditada que ayudará a asegurar el cumplimiento de los propósitos de la educación preescolar.

El aprendizaje incluye la observación, la manipulación de materiales, la relación causa y efecto, el desarrollo de los hechos y la búsqueda de soluciones para los variados problemas que se presentan. El cuestionamiento de la realidad circundante, la necesidad de descubrirla, conocerla, tomar contacto con ella, constituye el más sólido fundamento para la existencia de conjunto de actividades que abrirá ante el niño el camino del descubrimiento y la experimentación científica. Aproximarse, luego observar, más tarde experimentar, aseguran al niño un mayor conocimiento de la realidad, cuya organización y compresión se dará en un creciente orden de complejidad.

La dinámica del desarrollo es compleja, la formación de estructuras mentales requiere de una base emocional que anime al niño a actuar. La seguridad e independencia constituyen los elementos básicos para interactuar con el medio. La cooperación implica un proceso en el que intervienen aspectos afectivos-sociales, cognoscitivos y motores del desarrollo. Destaca la solución de problemas con responsabilidad, autovaloración, criticidad, cooperación y creatividad. Esto implica crear las condiciones ambientales y afectivas necesarias para que el niño construya el conocimiento (Del Niño y Maldonado, 2007).

Para Peralta (2007), el papel del docente en el Nivel Inicial en la enseñanza de los procesos de la ciencia debe ser el siguiente:

- Fomentar el desarrollo y práctica de los procesos cognitivos del alumno.

- Identificar los conocimientos previos que los alumnos tienen acerca del tema o contenido a enseñar, para relacionarlos con los que va a aprender.

- Hacer amena y atractiva la clase y tener en cuenta que el fin de su labor es que el alumno logre un aprendizaje significativo.

- Formular predicciones sobre el tema; plantear preguntas; aclarar dudas y resumir las ideas.

- Presentar el material de manera organizada, interesante y coherente. Intervenir con el objeto de mantener la discusión centrada y 
asegurar la utilización de las estrategias para que los alumnos las aprendan a utilizar.

\section{La experimentación para la promoción del desarrollo científico}

La experimentación representa la vía donde el niño aplica los conocimientos obtenidos, a través de la exploración, observación, análisis, creación de hipótesis y desarrollo de las habilidades relacionadas con el pensamiento analítico, crítico y creativo. Por este motivo, tal como lo señala Lacueva (2007): “...la experimentación resulta un elemento valioso para el docente ya que de una manera práctica, sencilla y atractiva encamina su quehacer educativo hacia la formación de habilidades de pensamiento más que a la mera acumulación de contenidos curriculares" (p. 34), de esta manera se logra, no solo la obtención de información de acuerdo a la materia estudiada, sino que además permitirá a los educandos resolver problemas del sistema en el que interactúan.

Cuando un maestro presenta un experimento al grupo, por más sencillo que este parezca, conducirá al desarrollo de determinados talentos en los estudiantes siempre y cuando la experimentación sea acompañada de cuestionamientos precisos y orientados hacia el análisis y la reflexión, según se van presentando y combinando los elementos que componen la actividad. Todo esto es un marco de diversión, curiosidad, respeto y juego, pues un experimento por si solo puede resultar provechoso para ilustrar un contenido académico.

El niño en la etapa preescolar está en la etapa idónea para adquirir habilidades reflexivas, por lo que el docente debe promover estrategias para hacerlo de forma efectiva. En este sentido, González (2012) refiere que: "En investigaciones realizadas, se concluye que el pensamiento reflexivo es una formación psicológica importante en la edad preescolar que ayuda a los niños a desarrollar habilidades en la etapa escolar". (p. 14). Por consiguiente, es menester de los educadores, activar esta habilidad lo cual es de suma importancia en la enseñanza de las ciencias y en el resto de las temáticas a abordar en la educación inicial.

Es así que el docente en esta etapa debe favorecer la experimentación científica en los niños, planificando y evaluando las actividades en función de los procesos de la ciencia, respetando la etapa de desarrollo de cada niño. Aunado a ello, debe ofrecer oportunidades para que los infantes exploren en función de lo que conocen, de manera surjan ideas y las relacionen con las ya existentes en su estructura cognitiva.

Por tal razón, el docente del nivel inicial debe ofrecer actividades de ciencia, motivando a sus educandos a explorar el mundo que lo rodea y a estimular en ellos, la inclinación hacia la ciencia, estimulando así la habilidad para la resolución de problemas efectivamente, empleando los procesos destinados para ello.

\section{MÉTODO}

La investigación se enmarcó en el paradigma cualitativo fundamentado en una investigación de campo de carácter descriptivo e interpretativo. Para Martínez (2009) "la investigación cualitativa consiste en: tratar de identificar, la naturaleza profunda de las realidades, su estructura dinámica, aquella que da razón plena de su comportamiento y manifestaciones" (p. 66).

El estudio se desarrolló bajo el enfoque cualitativo, porque se intentó conocer e interpretar las estrategias que aplica el docente de Educación Inicial para la promoción de la experimentación científica en los niños del Preescolar Ambrosio Plaza, ubicado en Guarenas, estado Miranda, Venezuela.

El diseño de la investigación fue el de una investigación de campo. Para Briones (1996), "la investigación de campo interpretativa exige ser cuidadosa y reflexiva para describir los acontecimientos cotidianos en el escenario de trabajo y para identificar el significado de las acciones de esos acontecimientos desde los 
diversos puntos de vista de los actores" (p. 199). El escenario estuvo representado por el Preescolar Ambrosio Plaza y los acontecimientos los llevados a cabo por las docentes.

Por otra parte, este trabajo responde a un carácter descriptivo e interpretativo, debido a que se registró la información ordenadamente del hecho investigado y se analizó posteriormente. En lo que se refiere a la investigación descriptiva comprende el registro, análisis e interpretación de la naturaleza actual y la composición a procesos de los fenómenos en estudio. (Tamayo y Tamayo, 2001). Por consiguiente, todos los datos recogidos en esta investigación fueron sometidos a un proceso descriptivo y de interpretación, con el fin de diseñar, aplicar y proponer estrategias de enseñanza dirigidas a la promoción de la experimentación científica en los niños del Preescolar Ambrosio Plaza.

Esta investigación se llevó a cabo en el Preescolar Ambrosio Plaza que funciona dentro de la Escuela Bolivariana del mismo nombre. Dicha institución está ubicada en la urbanización 27 de febrero en Guarenas, estado Miranda. Su infraestructura es de una planta física amplia, para atender una población de 1300 estudiantes. El preescolar está conformado por 3 aulas, se reciben niños desde los 3 años de edad hasta los 5 años. Cada salón tiene 1 maestra titular y 1 auxiliar, con 30 o 31 niños por aula. La población total del preescolar es de 92 niños y 6 maestras.

Es preciso recordar que los sujetos de la investigación se refiere al conjunto de personas, eventos, sucesos, comunidades, etc., sobre los cuales se habrán de recolectar los datos, sin que necesariamente sea representativo del universo o población que se estudia (Hernández et al., 2003).

Los sujetos de esta investigación fueron las 2 docentes titulares que laboraban en los grupos 1 y 2 del preescolar Ambrosio Plaza. Ambas con una experiencia entre 6 y 7 años de servicios ininterrumpidos y, por tanto, las responsables de la planificación y evaluación de los contenidos programáticos. También los 31 niños del grupo 1 se consideraron los sujetos a quienes se les aplicaron las estrategias diseñadas para promover en ellos la experimentación científica durante el año escolar 2016 - 2017.

En este trabajo se utilizó como técnica la observación, que implica el análisis, interpretación y la capacidad de describir los elementos que caracterizan la realidad percibida. Según Martínez (1998), la observación es "un conjunto de principios, normas y procedimientos metodológicos que permiten obtener conocimientos colectivos sobre una determinada realidad social" (p. 55). Esta se aplicó a las dos docentes titulares que laboran en los grupos 1 y 2 del preescolar, durante cuatro días continuos.

Los instrumentos para reportar las observaciones realizadas fueron una guía de observación y un cuaderno de registros diarios. La guía estuvo conformada por 8 aspectos a observar los cuales respondían a la clasificación de las estrategias de enseñanza propuestas por Díaz Barriga y Hernández (2009) en preinstruccional, coinstruccional y posinstruccional. En el cuaderno de registro se redactaron: a) las estrategias de enseñanza para la promoción de la experimentación científica que aplicaban las docentes titulares con los niños del preescolar, y b) los hechos observados durante la aplicación de las estrategias de enseñanza diseñadas por la autora de este estudio.

La investigación se estructuro en dos fases:

Fase I. Análisis de las estrategias de enseñanza: En esta fase, se procedió a observar a las docentes que laboraban en el nivel de Preescolar a fin de analizar las estrategias de enseñanza que empleaban en la promoción de la experimentación científica en los niños del Preescolar Ambrosio Plaza.

Fase II. Diseño de las estrategias. En esta fase, se procedió a realizar un arqueo bibliográfico para recoger mayores insumos y diseñar estrategias de enseñanza dirigidas a la promoción de la experimentación científica en los niños del Preescolar Ambrosio Plaza. 


\section{RESULTADOS}

\section{Fase I. Análisis de las estrategias de enseñanza}

Se procedió a realizar una observación durante dos días continuos, a fin de analizar la planificación y las estrategias empleadas por las docentes de los grupos 1 y 2 del Preescolar Ambrosio Plaza para las clases de ciencias. Se evidenció que ambas docentes no delimitaron la secuencia didáctica en inicio, desarrollo y cierre, lo cual indica que no parten de una planificación previa; por tanto, no hubo organización de las actividades a desarrollar. Los objetivos a impartir no estaban ni previa ni claramente definidos lo cual hizo pensar que no preparaban al niño en relación con la manera de cómo va a aprender, incidiendo en la activación o generación de conocimientos previos y ubicándolo en el contexto conceptual pertinente. Al no existir la organización de la secuencia los eventos, actividades y técnicas instruccionales quedan nulos.

En ambas docentes, se observó que desconocían la importancia de introducir variedad de actividades, medios y recursos para el desarrollo de las clases como fuentes de estímulos que motiven la curiosidad en los niños.

Finalmente, se concluyó que estos docentes no aplicaban estrategias de enseñanza ajustadas al deber ser de una secuencia instruccional que motiven a los niños de preescolar para el aprendizaje significativo de las ciencias. No tomaban en cuenta las necesidades ni los intereses de estos educandos para que ocurran en ellos construcciones conceptuales de la experimentación científica.

A partir de estos resultados reportados como deficientes en la planificación, aplicación y evaluación de estrategias de enseñanza, se procedió a diseñar un material instruccional ajustado a la experimentación científica en el nivel de preescolar dirigido a los docentes del Preescolar Ambrosio Plaza.

\section{Fase II. Diseño de las estrategias de enseñanza}

Para el diseño de las estrategias de enseñanza, se realizó una indagación teórica para sustentar la propuesta de promover la experimentación científica en los niños de preescolar. Para la presentación de las cinco estrategias propuestas se tomaron en cuenta los siguientes elementos: nombre de la estrategia, aprendizajes esperados, materiales necesarios, procedimientos a realizar, descripción del inicio, del desarrollo y el cierre de cada estrategia (ver Figura 1). 


\section{Estrategla 1}

HABITANTES MINÚSCULOS
Estraf*glas: 2

EN BUSCA DE LA ๒UE

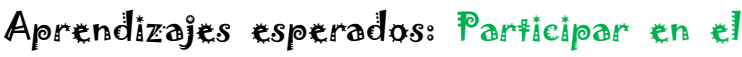

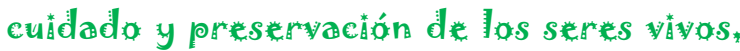
de su enforno famtlator escolar, comunîtarato.

Maferiales:

- 1 pafe de vídră

- 1 pala pequeña

- 1 frozo o pedazo de fela negra.

- 1 lìga (goma elásfüca)

- Un paco de fiterra.

- Medío vasa de agua.

- Azúcar.

- Trociános de frufa.

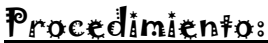

1- Se echa la fiterra en pofe de vildrito dejando un espación vacío en la parfe supertorto luego se infroducen en bofe unas cuantas hormïgas.

2- Se mezcla la fierra con frocî́fos de frufas. Se disuelven 5 a 6 cucharadas de azúcar en agua y se rocía la füerra del pafe con el agua azucarada.

3- Se fapa el pafe con la fela negra, sujefándola con la líga Se guarda la paf en un lugar oscuro y se espera un par de días.

4- Si las hormígas han fenído basfanfe oscuridad, habrían escavado fúneles junfo a las paredes de vídrio. Ello permât ver su hormïguero.
Aprendizajes Esperados:

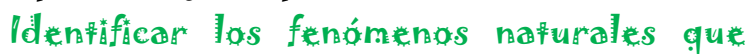
ocurren port la ünfluencỉa dẹl sol.

\section{Mafertideles:}

- I planfa (mafa) quu fenga un creciúmìnfo rápído y espígado.

- 1 caja de zapafos

- Carfulina

- Cunfa adhesiva

- Tỉjeras

\section{Procedämäenfo:}

1- Se recorfa un recuadro de carfulina que de lado mida aproximadamenfe la mä́ad de la anchura de la caja de zapafos que se ha elegito.

2- El recuadro de la cartulina se fija en

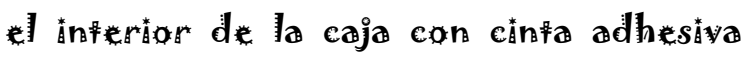
para que l recuadro quede bien adherido. 3- Se coloca la caja verfical y se hace un agujero con las fijeras en la parfe superíar. El agujero no debe ser demastado grande. Luego, se infroduce la planfa.

4- Se fapa la caja y se săfúa en un lugar donde haya mucha luz: No hay que olvildarse de quâtar la fapa para poder regar la planfa con un poco de agua. 
Inăcio: Se les presenfara a los esfudianfes, una lámina llusłrada con los confenídos a 7rabajar. Con fin de explatcarles con

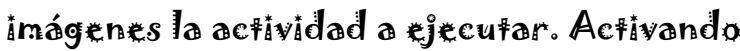

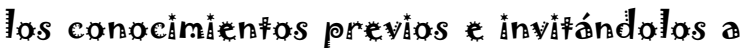
la formulacíón de pregunfas abiterfas; de acuerdo asu curiostadad.

Desarrollo: Lievar los núños al pafúlo exfertior ânvitararlos a observar las áreas verdes y pedirles que con una rastrillo pequeño, ctérnen la füerra y con una lupa busquen hormïgas y pregunfarles \& Cómo se comunäcan, cómo frabajan, cuál es color y el famaño de las mísmas? Con la ayuda y supervitsáón de la maesłra se van a Fransporta las hormïgas en el recípüenfe hacía el salón y proceder a realizar el hormïguero. Sollétartarles que se paren o se süenfen alrededor de la mesa y así puedan observar paso a pasa. Para luego dî́bujar fodo el procedímâtenfo.

Cierre: Luego de haber dibujado el proceso de ejecución. Se va a realizar una

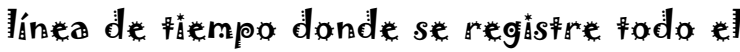
proceso evolufino, de dïcho hormíguero a fravés de âmágenés hechas par ellos mîsmos.

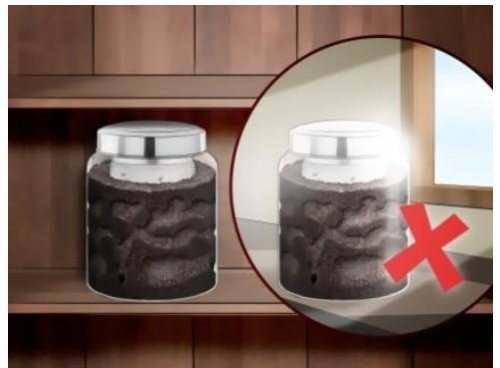

\section{Desarrollo de las estrafegías:}

Inäch̃o: En una ronda la maesfra conversará con los nîños, respefo valor de la nafuraleza. Que las planfas y los árboles son como un pulmón para nuesłro planéa. Que sĭn ellas; los anïmales y los seres humanos no podrían habïfar en la finerra.

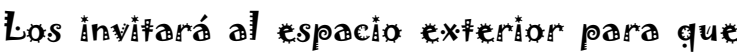
observen el huerfo escolar y puedan evildenciar que hay differenfes fípos de planfas, pero que fodas san ümportanfes para fodos los seres vavos de nuesforo planefa.

Desarrollo: Unïr dos mesas para presenfarle los maferiales a \#rabajar. Junfarlos alrededor de las mesas. Sollichifar la colaboractión de 2 nîños para que sürvan de asísfenfes y puedan pasar los maferiales de frabajo. Con la ayuda de la maestra se corfará el recuadro, para que la planfa

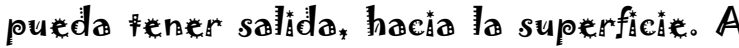
medila que se va desarrollando el procedî̀métenfo.

Cierre: enfregarles hojas blancas, lápiz y creyones. Para que dübujen fodo l proceso del \#rabajo elaborado.

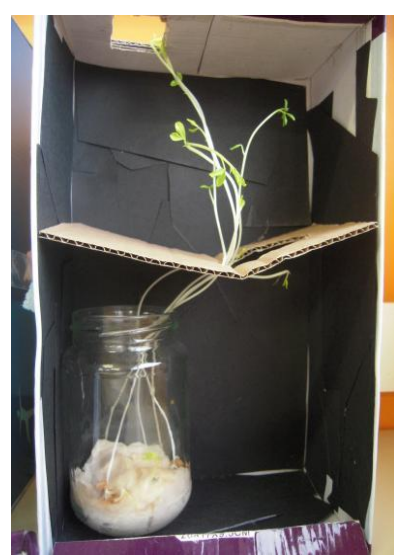




\section{Estraf*gla: 3 \\ \&POK QUE SALE MOHO EN EL PAN}

\section{Aprendizaje esperado:}

Formular algunas hẩpáf efectos en los experimenfos que realiza u abserva.

\section{Materiales:}

- 1 pafecon capa

- 1 paño con polva.

- Irebanada de pan duro

- 1 vasa con agua

\section{Procedimitenfo:}

1- Se infroduce la rebanada de pan en el vaso con agua. pasados unos segundos, se saca del vaso; anfes de que se humedezca demasiado.

2- Se sacude en laño con polvo sabre la rebanada de pan y se pone esfa denfro del pofe. Ino hay que olvidar cerrarlo! Al cabo de unas días, la rebanada cambía de aspecto.

\section{Desarrollo de la esfrafegia}

Inícỉa: En reuníón de grupo la maestra va a conversar con fodo el grupo, que no fodos los hongos fítenen el mismo famaño, que crecen al páte de los árboles y no fodos son comestâbles. Inväfándolos a descubrïr y asá poder desarrollar la actütadad.

\section{Estraf*gila: 4 \\ AGUA DULCE /AGUA SALADA}

\section{Aprendäzaje esperado:}

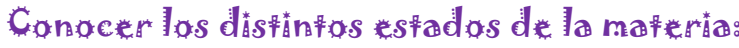

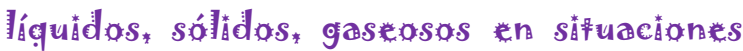
nafurales y de experìmentación sencillla.

\section{Maferatales:}

- Vjarra de agua.

- 1 cucharilla.

- 1vasa

- Coloranfe

- Trecípíenfe para los cubos de háelo

\section{Procedämäenfo:}

1- Se llena la jarra de agua y se añade el coloranfe. Hay que remover bäen con la cucharilla hasła conseguär que el agua quede coloreada. Se vierte la mezcla en los cubấfos y se pone en congelador.

2- Mintras se espera a que se congelen los cubä́los. Se llena lel vaso con agua. Luego se agregan 2 o 3 cucharadífas de sal. Se mezcla con energía para que la sal se dísuelva por complefó.

3- Cuando los cubä́los de hấlo esfén hechos, se refira uno del reciptente y se echa en lyaso de agua con sal.

4- Una vez desecho el cubífo, se abserva que agua dulce con coloranfe

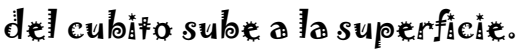


Desarrollo: Ubbícar al grupo en el espactio de experimentar y descubrìr y senfarlo alrededor de la mesa para que se ünfegren y parfäcüpen en la actüvĭdad. Se les debe explàcar que esfe experimento no es para comerlo, solo serviñá como abservacúón y experämenfacíán。

Cierre: Luega de ferminar el experimenfo,

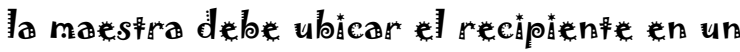
lugar vîsâlble pero lejos del alcance de los nâños. Es ämporfanfe recordar que fodo el proceso debe ser regísfrado, por los esłudianfes en hojas blancas.

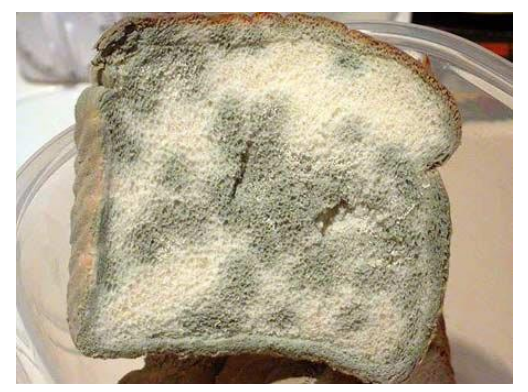

Desarrollo de las estrafegías:

Inăcha: En la ronda de bánenvenída la matsłra, les explicará a fodo el grupo que la mayor parfe del agua que cubre la fietra es salada. Pero la mísma no solo se le dîsfiñngue dell agua dulce por el sabor Invấándolos a realizzar el experimento y asá descubrir le fecto de las aguas junfas.

Desarrollo: Trasladar al grupo al comedor del colegía. Pedir una mesa y presenfar fodos los mafertales de frabajo y explicarles el procedînüenfo, para que ellos realicen fodo la proceso con la guía y ortenfacúón de la matesłra.

Cierre: Explicarle que lagua dulce el agua salada y el cubo de híelo del experañmfon represenfa las differenfes formas que puede adoptar lagua en planefa. La más abundanfe es el agua salada de los mares y océanos; mätenfras que l resfo es agua dulce dos ríos y lagos. El agua se hitela en el polo norfe y en el polo surr.

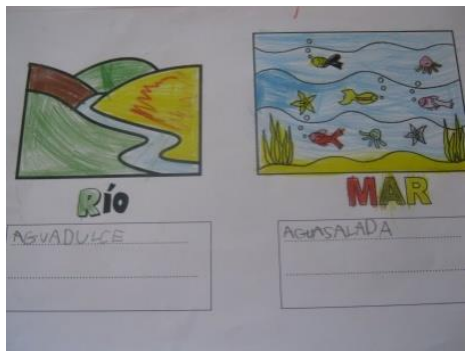




\section{Estrategia: 5}

FUEREA DE EMPUJE

\section{Aprendízaje sperado:}

Conocer el concepło de densildad y fuerza de empuje.

\section{Maferiales:}

- 3 vasas grandes

- Thuevo

- Agua

- Sal

- Azúcar

\section{Procedämátenfo:}

1. Identäficar con una túque cada vasa: uno con la efúquefa de azúcar, ofro de sal y afro de agua.

2. Se llenan con agua los \#res vasos has $¥$ la mâtad.

3. Al vaso con la palabra escrífa "sal" añadír una cucharada rebosada de sal y dìsolverla.

4. Al vasa con la palabra escrífa "azúcar" agregar una cucharada de esfe ängred dैente.

5. Colocar un huevo en lasa que fiten la palabra "agua" escrita. Observar que el huevo se hunde.

6. Luego sacar el huevo y colocarlo en el vaso con azúcar, observar qué sucede.

7 . Sacar el huevo y colocarlo en l vaso con sal, k̊quú sucéde?

8. Ahora en el vaso de agua salada agregar más agua nałural, oloservar qué sucéde.
Desarrollo de las estrafegías:

Inícǘ: En la ronda de büenvenída la maesłra, les explàcará a fodo el grupo que solor ll huevo actúan dos fuerzas: su peso y el empuje lla fuerza que ejerce hacia arriba el agua). Sa el pesa del huevo es mayar que el empuje del agua, el huevo se hundïrá. En caso confrarín, flofará sà el peso del huevo y el empuje del agua son íguales, el huevo quedará asá enfre dos aguas.

Al añadăr sal al agua logramos un láquädo más denso, lo que hace que empuje del huevo sea mayor.

Se ànvïła a los nîños a realizar el experimento y así descubrïr el efecto de las aguas.

Desarrollo: Trasladar al grupo al comedor del colegíd. Pedir una mesa y presenfar fodos los mafertales de frabajo y explatcarles el procedimitenfo; para que ellos realicen fodo el proceso con la guía y

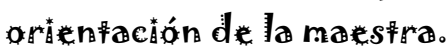

Ceterre: Expläcarles que esfe experimento nos muestra por quéc es más fácill flozar en agua de mar que en agua de rílos o pîscî́nas. La respuesła esłá en que el agua de mar, por la sal que pose es más densa que el agua del río o pấscîna.

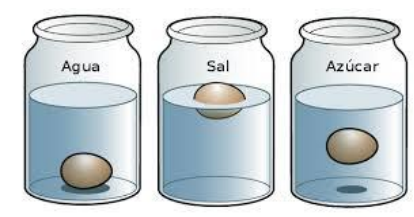




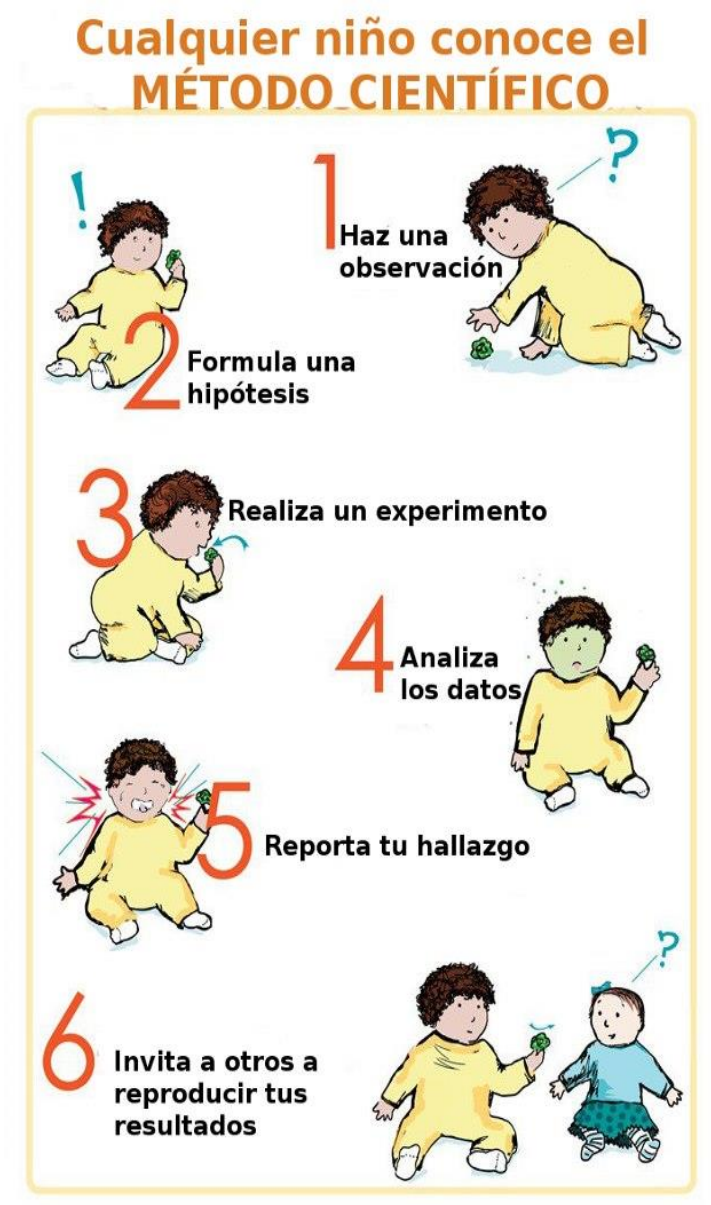

\section{CONCLUSIONES}

Las estrategias de enseñanza diseñadas mostraron una misma estructura metodológica de pedagogía activa con el fin de promover la exploración y experimentación del entorno natural de los niños del preescolar Ambrosio Plaza. Este diseño de estrategias facilitará a los niños acceder al conocimiento científico de manera significativa, contribuyendo así a mejorar la práctica educativa y fortaleciendo el desarrollo de las competencias científicas.

En el nivel de Preescolar, se requieren docentes bien formados y actualizados que asignen mayor atención a la experimentación científica con los niños entre 3 y 7 años de edad. Cobra pues importancia el diseño de estrategias innovadoras que aborden los aprendizajes esperados en esta área.

El análisis de las acciones de los niños en diversas situaciones experimentales brindará elementos de mayor profundidad para analizar la construcción de representaciones y nociones científicas que pudieran reflejarse en una enseñanza de las ciencias más adecuada para los niños en el preescolar.

\section{REFERENCIAS}

Alfonzo, A. (2003). Estrategias instruccionales. Trabajo no publicado. Caracas

Beltrán, L. (1996). Psicología de la educación. Madrid: Boixareu Universitaria

Briones, G. (1996). Metodología de la investigación cuantitativa en las ciencias sociales: Modulo 
Tres. Recuperado de http://www.icfes.gov.com/cont4/fomento//pu b/libros/ser_inv_soc/modulo3.pdf

Del Niño, Y. y Maldonado, R. (2007). Ciencias y desarrollo. España: Oficina de Educación Iberoamericana

Delors, J. (1997). La educación encierra un tesoro. Informe a la UNESCO de la Comisión Internacional sobre la Educación para el Siglo $X X I$

Díaz Barriga, F. y Hernández, G. (2009). Aprendizaje significativo: una interpretación constructivista. México: McGraw Hill

Díaz Barriga, F. y Hernández, G. (2010). Estrategias docentes para un aprendizaje significativo. Una interpretación constructivista. México: McGraw-Hill

Feo, R. (2010). Orientaciones Básicas para el Diseño De Estrategias Didácticas. Tendencias Pedagógicas, 16, 220 -236

Gallegos, L., Flores, F. y Calderón, E. (2008). Aprendizaje de las ciencias en preescolar: la construcción de representaciones y explicaciones sobre la luz y las sombras. En Revista Iberoamericana de Educación. 47, 97121

González, C. (2012). Promoción de la actividad reflexiva en preescolar. Típica Revista Electrónica. Recuperado de http://www.academia.edu/2061183/Promoci \%C3\%B3n_de_la_actividad_reflexiva_en_la_e dad_preescolar

Hernández, S., Fernández, C. y Batista, F. (2003). Metodología de la Investigación. México: Mc Graw Hill

Hurtado, J. (1998). Metodología de la Investigación. Caracas: Fundación Sypal

Lacueva, L. (2007). Las experiencias desencadenantes en Ciencia y Tecnología en la
Escuela. Madrid: Editorial Popular y Editorial Laboratorio Educativo

Martínez, M. (1998). La investigación cualitativa etnográfica. México: Trillas

Martínez, M. (2009). Ciencia y Arte en la Metodología Cualitativa. México: Trillas

Ministerio de Educación y Deportes. (2005). Educación Inicial, Bases curriculares. Caracas: Noriega

Ministerio de Educación y Deportes. (2007). Currículo de Educación Inicial. Caracas, Venezuela

Ministerio del Poder Popular para la Educación Inicial. (2014).Educación Inicial en Venezuela. Recuperado de http://www.me.gob.ve/noticias zonas/conteni do.php?id seccion $=16 \& i d$ contenido $=70 \& \mathrm{mo}$ do $=2$

Peralta, A. (2007). El aprendizaje por descubrimiento estimula el pensamiento lógico del niño en edad preescolar. (Trabajo de investigación). San Felipe, Venezuela: Autor

Rico, P. (2000). Hacia el perfeccionamiento de la escuela primaria. La Habana: Pueblo y Educación

Serrano, J. (2008). Estrategias para la enseñanza de las ciencias en niños de Educación Inicial del Jardín de Infancia Doctor José de Jesús Arocha (Trabajo de grado). Instituto Pedagógico de Miranda José Manuel Siso Martínez, Universidad Pedagógica Experimental Libertador (UPEL), Caracas, Venezuela

Tamayo, M. y Tamayo, L. (2001). El proceso de investigación científica. México: LIMUSA

Uría, M. (2001). Estrategia didáctica organizativa para mejorar los centros educativos. Madrid: Narcea S.A 